\title{
Imaging Case of the Month \\ Pilocytic Astrocytoma of the Cerebellopontine Angle in a Child Presenting With Auditory Neuropathy Spectrum Disorder
}

\author{
*Frederike Schneider, *Martin Kompis, †Christoph Ozdoba, $\ddagger$ Jürgen Beck, \\ *Marco Caversaccio, and *Pascal Senn \\ *University Department of Otorhinolaryngology, Head and Neck Surgery, †University Institute of Diagnostic \\ and Interventional Neuroradiology, and $\ddagger$ University Department of Neurosurgery, Inselspital, \\ Bern, Switzerland
}

Auditory neuropathy spectrum disorder (ANSD) is a clinical syndrome with hearing loss characterized by measurable otoacoustic emissions (OAEs) and absent or abnormal brain stem evoked response audiometry findings (BERA) $(1,2)$. Routine magnetic resonance imaging (MRI) has been advocated in children with ANSD because cochlear, neural, or central abnormalities are observed in up to $64 \%$ of affected cases (2). In the two largest reported imaging series comprising a combined total of 221 children, developmental malformations, such as cochlear nerve deficiency or hindbrain malformations, were predominantly observed, suggesting a benign origin of ASND in general. Bilateral ANSD cases are approximately 4 times more frequently associated with intracranial abnormalities compared with unilateral cases (2). In contrast, unilateral ANSD should remind the reader of tumor growth in the cerebellopontine angle (CPA), as previously reported in a single case (3) and with the presented case below.

\section{CASE PRESENTATION}

A 4-year-old male patient was referred to the speech and hearing therapy unit by his mother with the concerns of mild speech delay and a family history of speech disorders. The thorough clinical examination revealed minimal facial asymmetry with respect to eye and mouth muscular tone, House-Brackmann 1 and 2. Audiometric evaluation demonstrated left-sided, severe sensorineural hearing loss,

Address correspondence and reprint requests to Pascal Senn, M.D., Head of Cochlear Implant Division, University Department of Otorhinolaryngology, Head and Neck Surgery, Inselspital, 3010 Bern, Switzerland; E-mail: pascal.senn@insel.ch

The authors disclose no conflicts of interest. preserved transient evoked OAEs (TEOAEs), and pathologic BERA findings on the left side indicating unilateral ANSD (Fig. 1). On the right side, all tests were normal (Fig. 1).

The unenhanced, T2-weighted axial images showed a large, partially cystic, expansive tumor in the cerebellopontine angle (CPA) on the left side with displacement of the brain stem and the lower Cranial Nerves VII and VIII (Fig. 1). Postgadolinium axial and coronal sequences showed strong enhancement of the CPA lesion (Fig. 2).

A retromastoidal craniotomy with subtotal tumor removal was performed in the neurosurgery department. Total resection was not possible because of unclear borders between tumor mass and vital brain stem structures. Histopathology revealed a pilocytic astrocytoma Grade I. This rare, benign tumor occurs at a rate of 2 in 100,000 people and mostly in children or adolescents.

At 1 year after surgery, the child had stable hearing loss in the left ear, normal facial nerve function, and no other neurologic sequelae. Because of the residual tumor mass in the CPA, regular follow-up MRI scans will be administered. If tumor progression would be detected, revision surgery or radiosurgery could be evaluated as further therapeutic options.

\section{DISCUSSION AND CONCLUSION}

A tumor of the CPA is an unusual cause of ANSD in a child with unilateral hearing loss. Tumors of neuroepithelial origin like pilocytic astrocytomas, brainstem gliomas, medulloblastomas, or ependymomas are the most frequently observed tumors in the posterior fossa in childhood (4). These tumors may also grow or extend into the CPA; however, the differential diagnosis include vestibular schwannomas as the most common tumor of the CPA as well as meningiomas, epidermoids, atypical 


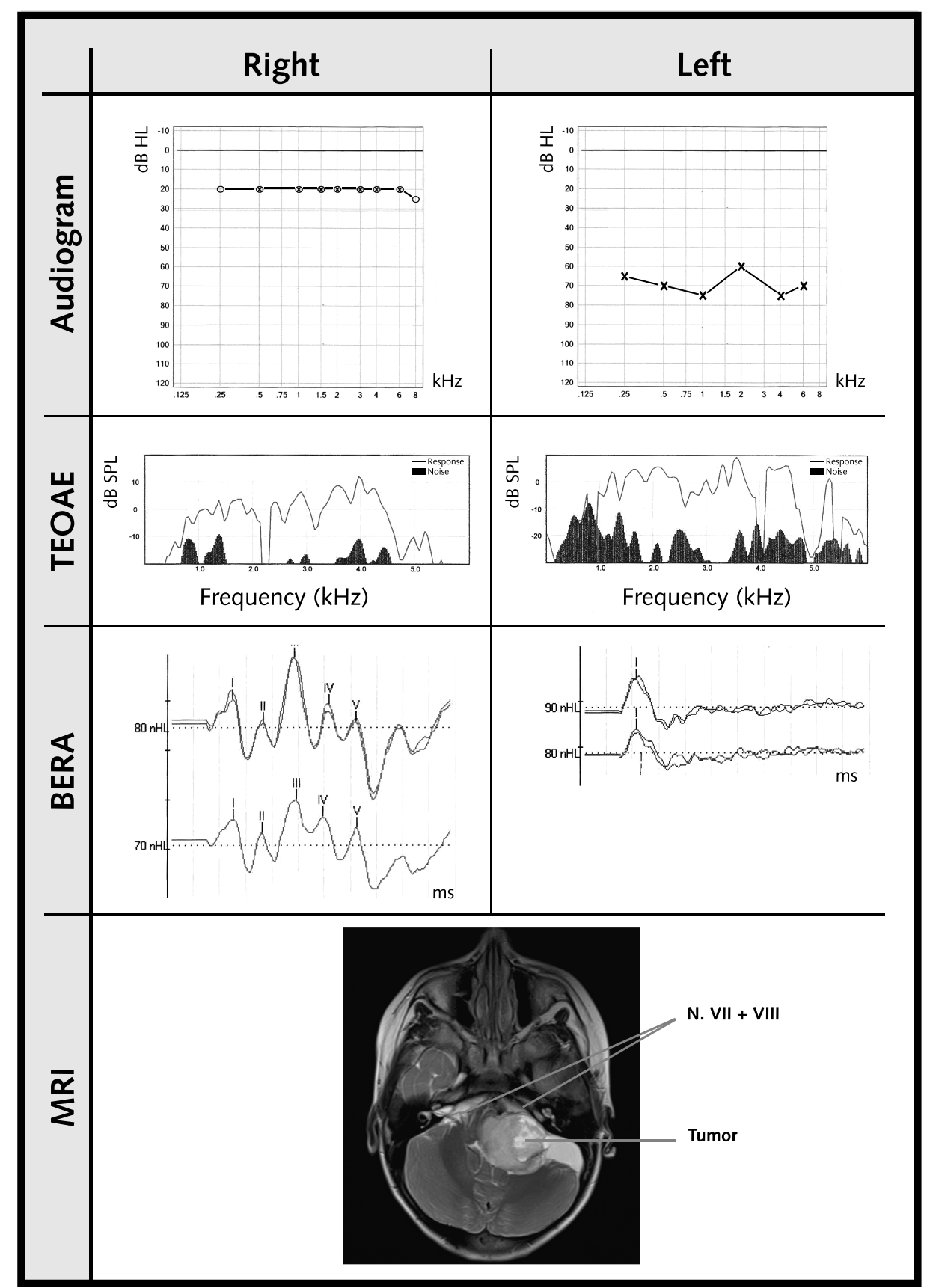

FIG. 1. Unilateral, left-sided auditory neuropathy spectrum disorder (ANSD) in a 4-year-old child with severe sensorineural hearing loss, preserved otoacoustic emissions (TEOAE), and abnormal brain stem evoked response audiometry (BERA). The unenhanced, T2-weighted MR image shows an expansive, partially cystic tumor in the left cerebellopontine angle with displacement of the brain stem and the lower cranial nerves VII and VIII.

teratoid/rhabdoid tumors, and germ cell tumors, among others. In the presented case with a large pilocytic astrocytoma in the left CPA, stretching or compression of the auditory nerve and mechanical stress on the nerve blood supply are the main mechanisms explaining the decreased neural function and the ANSD picture on the affected side. The partially preserved wave I in the BERA indicates some synchronous activity of spiral ganglion neurons; however, this signal could also contain cochlear microphonics because of a high variability of these measurements across ANSD cases (5). Nevertheless, in all cases of suspected or confirmed ANSD and particularly in unilateral cases, MRI and neuropediatric evaluation are highly recommended to rule out tumor growth in the CPA. The MRI examination protocol should include axial and sagittal unenhanced T1-weighted images, axial T2- 

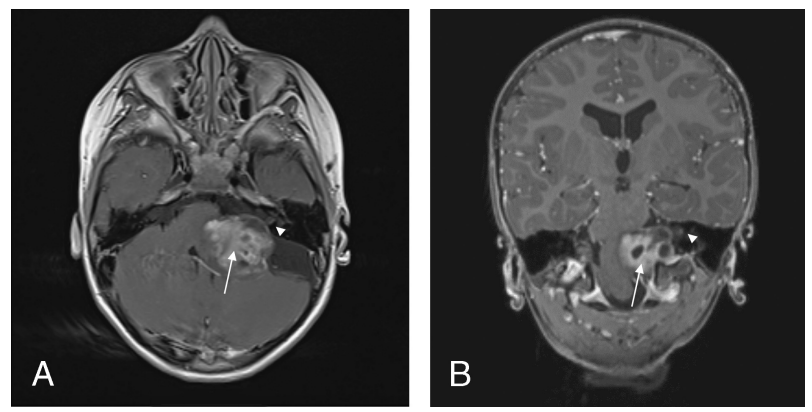

FIG. 2. Gadolinium-enhanced axial $(A)$ and coronary $(B)$ sequences show substantial contrast uptake of the tumor mass (arrow) in the left cerebellopontine angle with direct contact to the internal auditory canal (arrowhead).

weighted images, axial fluid-attenuated inversion recovery images of the entire brain, and a high-resolution 3D constructive interference in the steady state (CISS or SPACE more recently) sequence of the temporal bones. In case of tumor detection, contrast enhanced axial, coronary, and sagittal T1-weighted images should be acquired additionally.

\section{REFERENCES}

1. Buchmann CA, Roush A, Teagle HFB, et al. Auditory neuropathy characteristics in children with cochlear nerve deficiency. Ear Hear 2006;27:399-408.

2. Roche JP, Huang BY, Castillo M, et al. Imaging characteristics of children with auditory neuropathy spectrum disorder. Otol Neurotol 2010;31:780-8.

3. Nachman AJ. Retrocochlear hearing loss in infants: a case study of juvenile astrozytoma. Int J Audiol 2012;51:640-4.

4. Mirone G, Schiabello L, Chibbaro S, et al. Pediatric primary pilocytic astrocytoma of the cerebellopontine angle: a case report. Childs Nerv Syst 2009;25:247-51.

5. Starr A, Sininger Y, Nguyen T, et al. Cochlear receptor (microphonic and summating potentials, otoacoustic emissions) and auditory pathway (auditory brain stem potentials) activity in auditory neuropathy. Ear Hear 2001;22:91-9. 